\title{
Optical gyrotropy in quadrupolar Kondo systems
}

\author{
SungBin Lee, ${ }^{1}$ Arun Paramekanti, ${ }^{1,2}$ and Yong Baek Kim ${ }^{1,2,3}$ \\ ${ }^{1}$ Department of Physics, University of Toronto, Toronto, Ontario M5S 1A7, Canada \\ ${ }^{2}$ Canadian Institute for Advanced Research, Toronto, Ontario, M5G 1Z8, Canada \\ ${ }^{3}$ School of Physics, Korea Institute for Advanced Study, Seoul 130-722, Korea
}

(Dated: April 27, 2022)

\begin{abstract}
Recent experiments point to a variety of intermetallic systems which exhibit exotic quadrupolar orders driven by the Kondo coupling between conduction electrons and localized quadrupolar degrees of freedom. Using a Luttinger $k \cdot p$ Hamiltonian for the conduction electrons, we study the impact of such quadrupolar order on their energies and wave functions. We discover that such quadrupolar orders can induce a nontrivial Berry curvature for the conduction electron bands, leading to a nonvanishing optical gyrotropic effect. We estimate the magnitude of the gyrotropic response in a candidate quadrupolar material, $\mathrm{PrPb}_{3}$, and discuss the resulting Faraday rotation in thin films.
\end{abstract}

Kondo coupling between conduction electrons and local quadrupolar degrees of freedom is of great interest for realizing the multichannel Kondo lattice model. Candidate materials to realize this physics include Pr-based intermetallic compounds such as $\mathrm{PrPb}_{3}, \operatorname{Pr} T_{2} X_{20}$ (with $T=\mathrm{Ir}, \mathrm{Rh}, \mathrm{Ti}, \mathrm{V}$ and $X=\mathrm{Zn}, \mathrm{Al}), \operatorname{PrMg}_{3}, \operatorname{PrInAg} g_{2}$ and PrPbBi etc in which the quadrupoles reside on $4 f^{2} \mathrm{Pr}$ ions.[1-11] These ions possess a non-Kramers $\Gamma^{3}$ doublet ground state due to strong spinorbit coupling and local crystal fields. Matrix elements of the dipole operator, proportional to the total angular momentum, vanish in this doublet Hilbert space. However, matrix elements of quadrupolar operators, rank-2 irreducible tensors formed from the angular momentum, remain nonzero. The Doniach phase diagram suggests that strong hybridization between these quadrupolar doublets and the conduction electrons could lead to unusual heavy Fermi liquids, while weak hybridization could lead to quadrupolar orders driven by Ruderman-Kittel-Kasuya-Yosida (RKKY) interactions. [1215]

Detecting such quadrupolar orders and clarifying the nature of their broken symmetries remain challenging issues due to a dearth of probes which couple directly to the quadrupole moments. In contrast to magnetic dipole order, the ordering of these time-reversal invariant quadrupoles does not directly manifest itself in nuclear magnetic resonance (NMR), muon spin rotation $(\mu \mathrm{SR})$, or neutron diffraction measurements, necessitating the need for indirect probes. Such probes include: (i) ultrasonic measurements of phonon softening accompanying quadrupolar order, but this is restricted to ferroquadrupolar order; and (ii) magnetic field induced dipolar order, which can be probed by neutron diffraction and whose pattern depends on the underlying quadrupolar state, but this relies on having a field regime strong enough to induce measurable dipolar order while not significantly modifying the underlying quadrupolar order. $[6,16]$ This experimental complexity of probing multipolar orders is also at the heart of the longstanding puzzle of "hidden order" in $\mathrm{URu}_{2} \mathrm{Si}_{2}$. [17, 18]

In this Letter, we suggest an alternative route - the optical gyrotropic effect [19-22] - that may provide a sensitive probe of quadrupolar broken symmetries in metals. The optical gyrotropic effect is a certain handedness in the propa- gation of light, leading, for instance to one circular polarization of light propagating faster than the other. It may be observed in chiral states of matter such as a solution of chiral glucose molecules or materials with chiral charge order. In this Letter, we argue that quadrupolar Kondo systems naturally provide a broad class of materials in which to expect a nonzero gyrotropic effect. The underlying physics is simple to explain. Weak Kondo coupling of the quadrupoles to conduction electrons induces extended RKKY interactions between them. This frustration can lead to spiral quadrupolar order, which in turn, via the Kondo coupling, modifies the conduction electron dispersion and wave functions while preserving time-reversal symmetry. Such quadrupolar order breaks inversion and certain mirror symmetries, resulting in a nontrivial Berry curvature for the conduction electrons, and a nonzero gyrotropic response along certain high symmetry directions, measuring which can shed light on the nature of quadrupolar symmetry breaking.

As an illustrative example, we consider the intermetallic compound $\mathrm{PrPb}_{3}$ which has been suggested to exhibit spiral quadrupolar order below $T_{Q} \sim 0.4 \mathrm{~K}$.[23] In $\mathrm{PrPb}_{3}$, the Pr sites form a bipartite cubic lattice, so such spirals must arise from competing further-neighbor RKKY interactions which frustrate simple ferroquadrupolar order. To study the impact of this order on the conduction electrons, we consider the $k \cdot p$ Luttinger Hamiltonian with cubic symmetry,[24] which describes the spin-orbit coupled $\mathrm{Pb}$ conduction holes near the $\Gamma$-point. Such a Luttinger Hamiltonian is applicable to a wide variety of materials such as cubic intermetallics, GaAs, and the pyrochlore iridates.[24-26] We then incorporate new terms allowed by the broken symmetry associated with the weak quadrupolar order in $\mathrm{PrPb}_{3}$. Computing the Berry curvature of the resulting modified band wave functions is shown to lead to a nonzero gyrotropic effect. We estimate the magnitude of this gyrotropic response, discuss its possible signature in Faraday rotation experiments on thin films of $\mathrm{PrPb}_{3}$, and conclude with broader implications.

Luttinger Hamiltonian for conduction electrons. - We will consider conduction electrons with spin-orbit coupling, in the presence of time-reversal and cubic crystal symmetry. At the $\Gamma$-point, the point group symmetry is captured by the double 

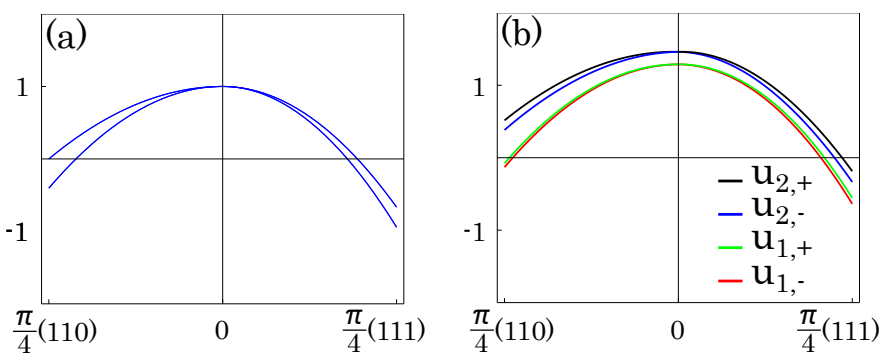

FIG. 1. Band dispersion (in eV) of the Luttinger Hamiltonian in Eq.(1), with momentum measured in units of the inverse lattice spacing. For both (a) and (b), two different momentum directions are shown: $\boldsymbol{k}$ along (110) and (111). (a) Doubly degenerate bands for the Luttinger Hamiltonian $\mathcal{H}_{0}$ with fitting parameters $\left(c_{1}, c_{2}, c_{3}\right) \sim(0.6,0.3,0.15) \mathrm{eV}$. (b) Band splitting due to the additional terms $\mathcal{H}_{Q}^{(1)}$ and $\mathcal{H}_{Q}^{(2)}$ with a choice $\alpha_{2} / \alpha_{1}=2, \alpha_{3} / \alpha_{1}=3$ and $\beta_{2} / \beta_{1}=\beta_{3} / \beta_{1}=-1$. The overall scale of these terms has been chosen to be significant in this figure, with $\alpha_{1}=\beta_{1}=0.05 \mathrm{eV}$, only in order to clearly depict the band splittings; for quantitative estimates of the gyrotropy in the paper, however, these are appropriately taken to be on the scale of the quadrupolar ordering temperature $T_{Q} \sim 0.4 \mathrm{~K}$. The eigenfunctions of $u_{1, \pm}$ and $u_{2, \pm}$ are given in Eq.(7).

group of $O_{h}$, which contains the maximal four-dimensional representation, the $\Gamma_{8}$ representation. Thus, electronic states near the $\Gamma$-point, for instance the $\mathrm{Pb}$ states of $\mathrm{PrPb}_{3}$, may be described by the widely applicable four-band Luttinger Hamiltonian,[24] $H_{0}=\sum_{\mathbf{k}} \Psi_{\mathbf{k} \mu}^{\dagger} h_{0}^{\mu \nu}(\mathbf{k}) \Psi_{\mathbf{k} \nu}$, with

$$
\begin{aligned}
h_{0}(\mathbf{k})= & -c_{1}\left(k_{x}^{2}+k_{y}^{2}+k_{z}^{2}\right) \mathbb{I}_{4}-c_{2}\left(k_{x}^{2} J_{x}^{2}+k_{y}^{2} J_{y}^{2}+k_{z}^{2} J_{z}^{2}\right) \\
& -c_{3}\left(k_{x} k_{y} K_{z}+k_{y} k_{z} K_{x}+k_{x} k_{z} K_{y}\right) .
\end{aligned}
$$

Here, $\mathbb{I}_{4}$ is the $4 \times 4$ identity matrix, $J_{\mu}$ is the $\mu$-th component of $j=3 / 2$ angular momentum matrices, and $K_{\mu}$ is defined via anticommutators, as $K_{x}=1 / 2\left\{J_{y}, J_{z}\right\}, K_{y}=$ $1 / 2\left\{J_{z}, J_{x}\right\}$, and $K_{z}=1 / 2\left\{J_{x}, J_{y}\right\}$. (Note that these angular momentum operators are distinct from the operators describing the local quadrupolar moments on Pr.) The dispersion is parameterized in terms of unknown constants $c_{1,2,3}$. Measuring the momentum in units of the inverse lattice constant $a$, we find that choosing $\left(c_{1}, c_{2}, c_{3}\right) \sim(0.6,0.3,0.15) \mathrm{eV}$ leads to a reasonable description of small hole pocket, as shown in Fig. 1(a), found near the $\Gamma$-point in $a b$ initio band structure studies of the closely related compound $\mathrm{LaPb}_{3} .[27,28]$ (We henceforth set $a=1$.) Time-reversal and inversion symmetry ensure that each band is doubly degenerate.

Quadrupolar ordering. - To understand the impact of quadrupole ordering on the conduction electrons, we follow a symmetry based approach which considers the modification of the above Luttinger Hamiltonian $H_{0}$ by additional terms which are allowed by the reduced symmetry of the quadrupolar ordered state. This enables us to directly connect the symmetry of the quadrupole ordering with the response of the electronic states.

To illustrate this idea, let us consider quadrupole order with $\boldsymbol{Q}=(Q, \pi, 0)$ that is known to be the ordering wave vector in $\mathrm{PrPb}_{3}$ (with $Q \approx 5 / 4 \pi$ ).[23] This ordering breaks translational symmetry, and the original cubic point group symmetry, but there are certain remnant symmetries: (i) $\mathcal{C}_{2 x}$ : two-fold rotations about the $\hat{x}$-axis, (ii) $\mathcal{M}_{x y}$ and $\mathcal{M}_{x z}$ : reflections in the mirror plane $x y$ or $x z$. Under these operations,

$$
\begin{aligned}
\mathcal{C}_{2 x} & :\left(k_{y}, k_{z}\right) \rightarrow\left(-k_{y},-k_{z}\right) ;\left(J_{y}, J_{z}\right) \rightarrow\left(-J_{y},-J_{z}\right)(2) \\
\mathcal{M}_{x z} & : k_{y} \rightarrow-k_{y},\left(J_{x}, J_{z}\right) \rightarrow\left(-J_{x},-J_{z}\right) \\
\mathcal{M}_{x y} & : k_{z} \rightarrow-k_{z},\left(J_{x}, J_{y}\right) \rightarrow\left(-J_{x},-J_{y}\right)
\end{aligned}
$$

Demanding that the modified Luttinger Hamiltonian be invariant under these residual symmetries, in addition to timereversal symmetry, leads to extra terms organized in powers of momentum,

$$
\begin{aligned}
& H_{Q}^{(1)}=\alpha_{1} J_{x}^{2}+\alpha_{2} J_{y}^{2}+\alpha_{3} J_{z}^{2} \\
& H_{Q}^{(2)}=\beta_{1} k_{y} J_{z}+\beta_{2} k_{z} J_{y}+\beta_{3} k_{x}\left(J_{x} J_{y} J_{z}+J_{z} J_{y} J_{x}\right) .
\end{aligned}
$$

As shown in Fig. 1(b), these terms split the two-fold band degeneracy of $\mathcal{H}_{0}$. For a different ordering wavevector $\boldsymbol{Q} \equiv$ $\left(Q_{1}, Q_{2}, 0\right)$, all cubic symmetries except $\mathcal{M}_{x y}$ are broken, leading to extra terms $\beta_{4} k_{x} J_{z}+\beta_{5} k_{z} J_{x}+\beta_{6} k_{y}\left(J_{x} J_{y} J_{z}+\right.$ $\left.J_{z} J_{y} J_{x}\right)$; this may be relevant to other materials.

We can compute the coefficients of these extra terms using the material-specific, symmetry allowed, Kondo couplings between the quadrupoles and the conduction electrons, and knowledge of the full bandstructure. On general grounds, since the quadrupoles are time-reversal invariant, they do not couple to the conduction electron spin, but rather to operators such as the local density or kinetic energy. For quadrupolar order at wavevector $\boldsymbol{Q}$, such Kondo couplings will couple unperturbed electronic states at momenta $(\mathbf{k}, \mathbf{k}+\boldsymbol{Q})$. Assuming that these states differ in energy by a characteristic energy scale $\Delta \varepsilon$, second order perturbation theory suggests that $\alpha_{i}, \beta_{i} \sim V^{2} / \Delta \varepsilon$, where $V$ is the strength of the Kondo coupling. More physically, since these terms arise below the quadrupolar transition temperature $T_{Q}$, which in turn is determined by the RKKY coupling $\sim V^{2} / \Delta \varepsilon$, we expect $\alpha_{i}, \beta_{i} \sim T_{Q}$.

We next study the impact of $H_{Q}^{(1)}, H_{Q}^{(2)}$ on the optical gyrotropy of the quadrupolar ordered state. Denoting the energy scale of the $k \cdot p$ Hamiltonian by $W$, two distinct momentum regimes emerge naturally: (i) small momentum, where $k \ll T_{Q} / W$, and (ii) large momentum, where $k \gg T_{Q} / W$. We next turn to an analytical perturbative approach to compute the Berry curvature and gyrotropic response arising from these regions of momentum space.

Small momentum, $k \ll T_{Q} / W$. - In this limit, we can start at the $\Gamma$-point where $H_{Q}^{(1)}$ breaks the four-fold degeneracy of the unperturbed states, leading to a pair of Kramers doublets. The leading corrections away from the $\Gamma$-point arise from linear-in-momentum terms present in $H_{Q}^{(2)}$, which weakly splits these Kramers pairs.

For the $(Q, \pi, 0)$ order relevant to $\mathrm{PrPb}_{3}$, projecting to the eigenstates of $H_{0}+H_{Q}^{(1)}$ at the $\Gamma$-point, only terms $\beta_{1}, \beta_{2}$ in $H_{Q}^{(2)}$ have nonzero matrix elements, while the matrix element 
of the $\beta_{3}$ term vanishes. The splitting of each doublet away from the $\Gamma$-point can then be described in terms of just two Pauli matrices, leading to a vanishing Berry curvature over any small momentum patch. Along $k_{y}=k_{z}=0$, the doublet remains unsplit, leading to a line node, and the Berry curvature is not well-defined on a patch which intersects this line node. However, the gyrotropic response, as discussed below, integrates the Berry curvature over all occupied bands; thus, patches near the line node also do not contribute to the final result since the contributions from the two bands touching at the line node will mutually cancel. Thus, we expect the small momentum region gives a vanishing contribution to the gyrotropy in $\mathrm{PrPb}_{3}$. Note, however, that for more general $\left(Q_{1}, Q_{2}, 0\right)$ order, this is no longer true.

Large momentum, $k \gg T_{Q} / W$. - In this limit, we first diagonalize $\mathcal{H}_{0}$ (see Supplemental Material for energies and eigenfunctions) which leads to two pairs of doubly degenerate bands, and project $H_{Q}^{(1)}$ and $H_{Q}^{(2)}$ into each degenerate manifold. The projection of $H_{Q}^{(1)}$ turns out to be proportional to the identity matrix, and hence does not modify the eigenstates which remain degenerate. We thus focus on the effects of $H_{Q}^{(2)}$, and express its projection into each doublet of $\mathcal{H}_{0}$ using Pauli matrices, as $\tilde{\mathcal{H}}_{\ell}=\vec{f}_{\ell}(\boldsymbol{k}) \cdot \vec{\sigma}$, where $\ell=1,2$ labels the two different degenerate bands. The eigenvalues are given by $E_{\ell, \pm}(\mathbf{k})=\epsilon_{\ell}(\mathbf{k}) \pm\left|\vec{f}_{\ell}(\mathbf{k})\right|$, where $\epsilon_{\ell}(\boldsymbol{k})$ are the unperturbed (degenerate) band energies, and the eigenfunctions are

$$
u_{\ell, \pm}(\mathbf{k})=\left(\begin{array}{c} 
\pm \sqrt{\frac{1}{2} \pm \frac{f_{\ell, x}(\mathbf{k})}{2\left|\vec{f}_{\ell}(\mathbf{k})\right|}} \\
\frac{f_{\ell, x}(\mathbf{k})+i f_{\ell, y}(\mathbf{k})}{\sqrt{2\left(\left|\overrightarrow{\vec{f}_{\ell}}(\mathbf{k})\right|^{2} \pm f_{\ell, z}(\mathbf{k})\left|\overrightarrow{f_{\ell}}(\mathbf{k})\right|\right)}}
\end{array}\right) .
$$

Fig. 1 (b) illustrates how doubly degenerate bands in Fig.1 (a) split into four bands with eigenfunctions $u_{\ell, \pm}(\boldsymbol{k})$ in the presence of $\mathcal{H}_{Q}^{(2)}$, for given parameters $\alpha_{2} / \alpha_{1}=2, \alpha_{3} / \alpha_{1}=$ 3 and $\beta_{2} / \beta_{1}=\beta_{3} / \beta_{1}=-1$. The overall scale of these terms has been chosen to be significant in Fig. 1(b), with $\alpha_{1}=$ $\beta_{1}=0.05 \mathrm{eV}$, only in order to clearly depict the band splittings; however, for quantitative estimates of the gyrotropy discussed below, these are correctly taken to be on the scale of $T_{Q} \sim$ $0.4 \mathrm{~K}$. For given eigenfunctions say $u_{\ell, \pm}$, the $\gamma$ component of Berry curvatures $\Omega_{\ell, \pm}^{\gamma}$ are defined as

$$
\Omega_{\ell, \pm}^{\gamma}=i \epsilon_{\mu \nu \gamma}\left\langle\partial_{k_{\mu}} u_{\ell, \pm} \mid \partial_{k_{\nu}} u_{\ell, \pm}\right\rangle,
$$

where $\epsilon_{\mu \nu \gamma}$ is the totally antisymmetric tensor. A nonzero Berry curvature $\Omega_{\ell, \pm}^{\gamma}$ induces an anomalous velocity of conduction electrons parallel to $\mu$ direction when the electric field is applied along $\nu$ direction. In the time reversal invariant but lattice symmetry broken system, such anomalous velocity induces the transverse current $j_{\mu}=\lambda_{\mu \nu \gamma}^{G} d E_{\nu} / d x_{\gamma}$ that is proportional to the gyrotropic coefficient $\lambda_{\mu \nu \gamma}^{G}$. In particular, for oscillating electric field $E_{\nu}\left(x_{\gamma}, t\right)=\operatorname{Re}\left[E e^{i\left(q x_{\gamma}-\omega t\right)}\right]$ that propagates along the $\gamma$ direction with wave vector $q$ and frequency $\omega$, the gyrotropic coefficient $\lambda_{\mu \nu \gamma}^{G}$ can be derived within the relaxation time approximation,[22]

$$
\lambda_{\mu \nu \gamma}^{G} \approx \frac{e^{2}}{\hbar} \frac{2}{(2 \pi)^{3}} \frac{l_{m f}}{(1-i \omega \tau)^{2}} \int_{k_{\gamma}>0} d k_{\gamma} \Phi^{\gamma}\left(k_{\gamma}\right),
$$

for $q l_{m f} \ll 1$ where $l_{m f}=v_{F} \tau$ is a mean free path with Fermi velocity $v_{F}$ and relaxation time $\tau$, and $\Phi^{\gamma}\left(k_{\gamma}\right) \equiv$ $\int_{o c c} d k_{\mu} d k_{\nu} \Omega^{\gamma}(\boldsymbol{k})$ is the 2D integral of Berry curvatures for a given $k_{\gamma}>0$. The detailed derivation of $\lambda_{\mu \nu \gamma}^{G}$ in Eq.(9) is shown in the Supplemental Material.

We begin by discussing how symmetry constrains the gyrotropic effect. The $\gamma$ component of Berry curvature is odd under the mirror symmetries if a mirror plane contains $\gamma$ direction. Therefore, for quadrupole order with the wave vector $\boldsymbol{Q}=(Q, \pi, 0)$, since mirror symmetries $\mathcal{M}_{x y}$ and $\mathcal{M}_{x z}$ are present, this guarantees $\Phi^{x}\left(k_{x}\right)=\Phi^{y}\left(k_{y}\right)=\Phi^{z}\left(k_{z}\right)=0$. Thus, we expect $\lambda_{\mu \nu \gamma}^{G}=0$ for $\gamma=[100]$, [110] directions. However, there are no mirror planes for $\gamma=$ [111], so we expect $\Phi^{\gamma}\left(k_{\gamma}\right) \neq 0$ for this direction.

To estimate the gyrotropy for $\gamma=$ [111], we compute the 2D integration of Berry curvatures $\Phi^{\gamma}\left(k_{\gamma}\right)$ including all four bands, based on the eigenvalues and eigenfunctions of $\tilde{\mathcal{H}}_{\ell}$. For a spherical hole pocket, one can further simplify the integration into only angle dependence, realizing that $\int d k_{\gamma} \Phi^{\gamma}\left(k_{\gamma}\right)$ does not depend on the Fermi wave vector in the limit of $k \gg T_{Q} / W$. Now, we consider the surface integration of $\Phi^{\gamma}\left(k_{\gamma}\right)$ in Eq.(9)

$$
\int_{k_{\gamma}>0} d k_{\gamma} \Phi^{\gamma}\left(k_{\gamma}\right) \approx \frac{1}{W} \int_{\theta, \varphi}\left(\left|\overrightarrow{f_{1}}\right| \Omega_{1,+}^{\gamma}+\left|\overrightarrow{f_{2}}\right| \Omega_{2,+}^{\gamma}\right)(10)
$$

Here, the integration of azimuthal and polar angles $\varphi$ and $\theta$ for upper half of a sphere is $\int_{\theta, \varphi}=\int_{0}^{\pi / 2} \sin \theta d \theta \int_{0}^{2 \pi} d \varphi$, where $\boldsymbol{k}$ is defined as $\left(k_{\mu}, k_{\nu}, k_{\gamma}\right)=k(\sin \theta \cos \varphi, \sin \theta \sin \varphi, \cos \theta)$ in spherical coordinates. (See the Supplemental Material for a detailed derivation of Eq.(10).)

Fig. 2 (a) and (b) show the azimuthal and polar angle dependence of $\left|\overrightarrow{f_{1}}\right| \Omega_{1,+}^{\gamma}$ and $\left|\overrightarrow{f_{2}}\right| \Omega_{2,+}^{\gamma}$ (in units of $T_{Q}$ ) respectively for $c_{2}=0.3 \mathrm{eV}, c_{3}=0.15 \mathrm{eV}$ (results do not depend on $c_{1}$ ) and $\beta_{1}=-\beta_{2}=-\beta_{3}=T_{Q}$. One can easily confirm that $\left|\vec{f}_{\ell}\right| \Omega_{\ell,+}^{\gamma}$ is odd under time reversal symmetry : $\theta \rightarrow \pi-\theta$ and $\varphi \rightarrow \pi+\varphi$. Integration of upper half of a sphere for both $\left|\overrightarrow{f_{\ell}}\right| \Omega_{\ell,+}^{\gamma}$ results in $\int_{\theta, \varphi}\left(\left|\overrightarrow{f_{1}}\right| \Omega_{1,+}^{\gamma}+\left|\overrightarrow{f_{2}}\right| \Omega_{2,+}^{\gamma}\right)=\eta T_{Q}$, with the coefficient $\eta \sim 0.1-1$ depending on the relative signs of $\beta_{1}, \beta_{2}$ and $\beta_{3}$.

Experimental Signature. - So far, we have considered how quadrupole order can induce band Berry curvature and a nonzero gyrotropic coefficient $\lambda_{\mu \nu \gamma}^{G}$. Such gyrotropy will lead to a finite Faraday rotation of light transmitted through thin films with quadrupolar order. Including the gyrotropic coefficient $\lambda_{\mu \nu \gamma}^{G}$, the conductivity tensor can be represented as $\sigma_{\mu \nu}(\omega, k) \simeq \sigma(\omega) \delta_{\mu \nu}+i \lambda_{\mu \nu \gamma}^{G} k_{\gamma}$. Using Maxwell equations $\nabla \times \boldsymbol{B}=\mu_{0} \boldsymbol{j}$ and $\nabla \times \boldsymbol{E}=-\frac{\partial \boldsymbol{B}}{\partial t}$ with vacuum permeability $\mu_{0}$, the complex index of refraction $N \equiv c k / \omega$ ( $c$ is the speed of light) can be written up to the leading order of $\lambda_{\mu \nu \gamma}^{G}$ $: N_{ \pm} \approx(n+i \kappa) \pm i \mu_{0} c \lambda_{\mu \nu \gamma}^{G} / 2$ for right- or left-circularly 

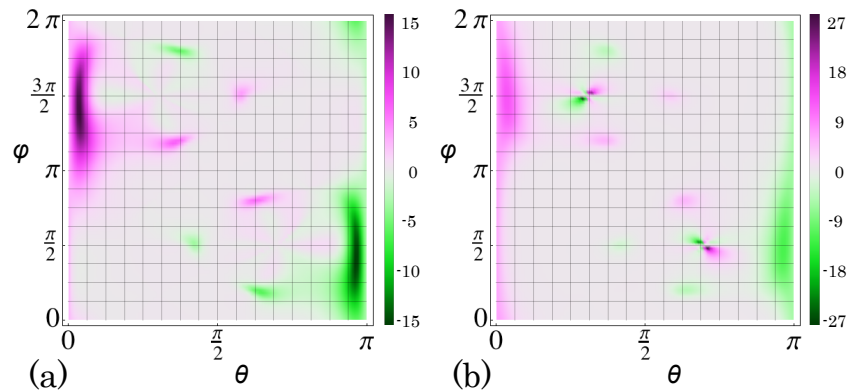

FIG. 2. Azimuthal and polar angle dependence of $\left|\vec{f}_{1}\right| \Omega_{1,+}^{\gamma}$ and $\left|\overrightarrow{f_{2}}\right| \Omega_{2,+}^{\gamma}$ in units of $T_{Q}$, with given parameters $c_{2}=0.3 \mathrm{eV}$, $c_{3}=0.15 \mathrm{eV}$ (these results are independent of $c_{1}$ ) and $\beta_{1}=-\beta_{2}=$ $-\beta_{3}=T_{Q}$ when $\gamma=[111]$. See the main text for details.

polarized electric fields $\boldsymbol{E}=\left(\hat{x}_{\mu} \pm i \hat{x}_{\nu}\right) E_{0} e^{i\left(k x_{\gamma}-\omega t\right)}$, where $n+i \kappa$ is the normal complex refractive index in the absence of gyrotropy. This difference of refractive indices leads to the rotation of the principal axis polarization of incident light, with the Faraday rotation angle $\theta_{F}$ given by

$$
\begin{aligned}
\theta_{F} & =\frac{\omega d}{2 c} \operatorname{Re}\left[N_{+}-N_{-}\right] \approx \frac{\omega d}{2 c} \operatorname{Im}\left[\mu_{0} c \lambda_{\mu \nu \gamma}^{G}\right] \\
& \approx \frac{\omega d}{2 c} \frac{\alpha l_{m f}}{\pi^{2}} \operatorname{Im}\left[\frac{1}{(1-i \omega \tau)^{2}}\right] \int_{k_{\gamma}>0} d k_{\gamma} \Phi^{\gamma}\left(k_{\gamma}\right),
\end{aligned}
$$

where we have used Eq.(9) for the gyrotropic coefficient $\lambda_{\mu \nu \gamma}^{G}$. Here, $d$ is the film thickness of materials, $\alpha=e^{2} /\left(4 \pi \epsilon_{0} \hbar c\right)$ is the fine structure constant, and $l_{m f}=v_{F} \tau$ is the mean free path determined by the Fermi velocity $v_{F}$ and the transport lifetime $\tau$. Next, let us estimate the Faraday rotation angle per unit thickness for $\mathrm{PrPb}_{3}$ films. To maximize $\theta_{F} / d$, we need to work at frequencies $\omega \sim 1 / \tau$, which leads to

$$
\frac{\theta_{F}}{d} \sim \frac{\alpha}{2 \pi^{2}} \frac{v_{F}}{c} \int_{k_{\gamma}>0} d k_{\gamma} \Phi^{\gamma}\left(k_{\gamma}\right),
$$

the estimate of which depends the Fermi velocity $v_{F}$ and the integrated Berry curvature.

(1) Fermi velocity and transport lifetime. - The electronic bands have been studied for $\mathrm{LaPb}_{3}$ where $\mathrm{La}^{3+}$ forms closed shell and is inert without quadrupolar order from $\mathrm{Pr}^{3+}$ $4 f^{2}$ non-Kramers doublet. It shows the hole pockets near $\Gamma$ point in $\mathrm{LaPb}_{3}$ due to the conduction electrons of $\mathrm{Pb}$, which have been also observed for $\mathrm{PrPb}_{3}$ in de Haas-van Alphen experiments.[27-29] Strong spin-orbit coupling of $\mathrm{Pb}$ conduction electrons suggests that the $\mathrm{Pb}$ electronic states are well described by the Luttinger Hamiltonian $\mathcal{H}_{0}$ in Eq.(1). Based on the band structure calculation of $\mathrm{LaPb}_{3}$, we estimate the Fermi velocity $v_{F} \approx 2 \times 10^{6} \mathrm{~m} / \mathrm{s}$.

Furthermore, we obtain the carrier density $n_{d}=4 \times$ $k_{F}^{3} /\left(6 \pi^{2}\right) \approx 2.5 \times 10^{26} \mathrm{~m}^{-3}$ arising from the four hole pocket bands with $k_{F} a \approx \pi / 4$ (lattice constant $a \sim 5 \AA$ ), with an effective mass $m^{*} \approx 0.1 m_{e}$. Within a Drude picture, the complex conductivity is written as $\sigma(\omega)=\sigma_{0} /(1-i \omega \tau)$, where $\sigma_{0}=n_{d} e^{2} \tau / m^{*}$. Close to the quadrupole ordering temperature $T_{Q}, \sigma_{0} \approx 1 \mu \Omega^{-1} \mathrm{~cm}^{-1}$ for $\mathrm{PrPb}_{3}$, which yields $\tau \approx 10^{-12} s$. We thus need to probe the gyrotropy at a frequency $\omega \approx 10^{12} \mathrm{~Hz}$.

(2) Integrated Berry curvature. - As mentioned earlier, $\mathrm{PrPb}_{3}$ exhibits spiral quadrupolar order at $T_{Q} \approx 0.4 \mathrm{~K}$ with an ordering wave vector $\boldsymbol{Q} \approx(Q, \pi, 0)$ where $Q \approx 5 / 4 \pi$. Based on our previous discussion, the integrated Berry curvature from the reconstructed bands is $\int_{k_{\gamma}>0} d k_{\gamma} \Phi^{\gamma}\left(k_{\gamma}\right) \approx \eta T_{Q} / W$ (with $\eta \sim 0.1-1$ ). This leads to the final result

$$
\frac{\theta_{F}}{d} \sim \frac{\alpha}{2 \pi^{2} a}\left(\frac{v_{F}}{c}\right)\left(\frac{\eta T_{Q}}{W}\right) .
$$

where we have reinstated the lattice constant $a$.

(3) Faraday angle. - Since $\mathrm{PrPb}_{3}$ is metallic, we need to estimate the optimal film thickness for which we expect significant transmission of light, with the maximum possible Faraday angle. The penetration depth $\delta$ of light is determined by the complex refractive index $N=n+i \kappa$ via $\delta=c / \omega \kappa$. For $\omega \approx 10^{12} \mathrm{~Hz}$ (i.e., infrared light), and a complex Drude conductivity with $\sigma(\omega)=\sigma^{\prime}(\omega)+i \sigma^{\prime \prime}(\omega)$, we find $\kappa \approx \sqrt{\mu_{0} c^{2} \sigma^{\prime}(\omega) / \omega} \approx 3 \times 10^{3}$, so the estimated penetration depth is $\delta=c / \omega \kappa \approx 100 \mathrm{~nm}$, and we must choose the film thickness $d \sim \delta$.

Thus, for $T<T_{Q}$, when the infrared light with a frequency $\omega \approx 10^{12} \mathrm{~Hz}$ is transmitted through $\mathrm{PrPb}_{3}$ along the [111] direction, setting $\eta \gtrsim 0.1$ yields a Faraday rotation angle $\theta_{F} \gtrsim 0.01 \mu \mathrm{rad}$ for a film thickness $d \approx 100 \mathrm{~nm}$. Such small rotation angles have been measured in Kerr experiments on superconducting materials at very low temperatures, so we expect the Faraday rotation to be accessible in future experiments. [30, 31]

Conclusion. - Motivated by a variety of intermetallic systems that exhibit quadrupolar orders, we have proposed that optical gyrotropy in quadrupolar Kondo systems [3, 4, 7$10,32]$ may yield further information on the nature of broken symmetry. Kondo coupling between conduction electrons and localized quadrupolar moments can naturally drive interesting quadrupolar order and further induce non-trivial Berry curvature for conduction electrons. Such effect leads to a certain handedness of light propagation, resulting in Faraday rotation. Using a Luttinger $k \cdot p$ Hamiltonian for conduction electrons, we explored how quadrupolar order impacts the gyrotropy. Finally, we estimated the Faraday rotation angle for a candidate material $\mathrm{PrPb}_{3}$, which can be measured in future experiments. The optical gyrotropic effect might thus serve to shed further light on "hidden" quadrupolar orders.

We are grateful to S. Bhattacharjee, V. S. Venkataraman and J. Orenstein for useful discussions. We acknowledge support from NSERC of Canada (SBL, YBK, AP) and the Center for Quantum Materials at University of Toronto (SBL, YBK). 
[1] A. Andreeff, E. A. Goremychkin, H. Griessmann, B. Lippold, W. Matz, O. D. Chistyakov, and E. M. Savitskii, physica status solidi (b) 98, 283 (1980).

[2] R. Galera, A. Murani, and J. Pierre, Journal of Magnetism and Magnetic Materials 23, 317 (1981).

[3] P. Morin, D. Schmitt, and E. Du Tremolet de Lacheisserie, Journal of Magnetism and Magnetic Materials 30, 257 (1982).

[4] M. Giraud, P. Morin, and D. Schmitt, Journal of Magnetism and Magnetic Materials 52, 41 (1985).

[5] H. Suzuki, M. Kasaya, T. Miyazaki, Y. Nemoto, and T. Goto, Journal of the Physical Society of Japan 66, 2566 (1997).

[6] H. Tanida, H. S. Suzuki, S. Takagi, H. Onodera, and K. Tanigaki, Journal of the Physical Society of Japan 75, 073705 (2006).

[7] T. Onimaru, K. T. Matsumoto, Y. F. Inoue, K. Umeo, T. Sakakibara, Y. Karaki, M. Kubota, and T. Takabatake, Phys. Rev. Lett. 106, 177001 (2011).

[8] T. Onimaru, N. Nagasawa, K. T. Matsumoto, K. Wakiya, K. Umeo, S. Kittaka, T. Sakakibara, Y. Matsushita, and T. Takabatake, Phys. Rev. B 86, 184426 (2012).

[9] A. Sakai and S. Nakatsuji, Journal of the Physical Society of Japan 80, 063701 (2011).

[10] A. Sakai, K. Kuga, and S. Nakatsuji, Journal of the Physical Society of Japan 81, 083702 (2012).

[11] K. Iwasa, H. Kobayashi, T. Onimaru, K. T. Matsumoto, N. Nagasawa, T. Takabatake, S. Ohira-Kawamura, T. Kikuchi, Y. Inamura, and K. Nakajima, Journal of the Physical Society of Japan 82, 043707 (2013), http://dx.doi.org/10.7566/JPSJ.82.043707.

[12] Q. Si and F. Steglich, Science 329, 1161 (2010).

[13] D. L. Cox, Phys. Rev. Lett. 59, 1240 (1987).

[14] D. L. Cox and M. Jarrell, Journal of Physics: Condensed Matter 8, 9825 (1996).

[15] P. Coleman, Heavy fermions: Electrons at the edge of magnetism (Wiley Online Library, 2007).

[16] T. Onimaru, T. Sakakibara, N. Aso, H. Yoshizawa, H. S. Suzuki, and T. Takeuchi, Phys. Rev. Lett. 94, 197201 (2005).

[17] T. T. M. Palstra, A. A. Menovsky, J. v. d. Berg, A. J. Dirkmaat, P. H. Kes, G. J. Nieuwenhuys, and J. A. Mydosh, Phys. Rev. Lett. 55, 2727 (1985).

[18] P. Chandra, P. Coleman, and R. Flint, Nature 493, 621 (2013).
[19] L. D. Landau, J. Bell, M. Kearsley, L. Pitaevskii, E. Lifshitz, and J. Sykes, Electrodynamics of continuous media, Vol. 8 (Elsevier, 1984).

[20] A. R. Bungay, Y. P. Svirko, and N. I. Zheludev, Phys. Rev. B 47, 11730 (1993).

[21] P. Hosur, A. Kapitulnik, S. A. Kivelson, J. Orenstein, and S. Raghu, Phys. Rev. B 87, 115116 (2013).

[22] J. Orenstein and J. E. Moore, Phys. Rev. B 87, 165110 (2013).

[23] T. Onimaru, T. Sakakibara, N. Aso, H. Yoshizawa, H. Suzuki, and T. Takeuchi, Physical review letters 94, 197201 (2005).

[24] J. M. Luttinger, Phys. Rev. 102, 1030 (1956).

[25] B.-J. Yang and Y. B. Kim, Phys. Rev. B 82, 085111 (2010).

[26] E.-G. Moon, C. Xu, Y. B. Kim, and L. Balents, Phys. Rev. Lett. 111, 206401 (2013).

[27] S. Ram, V. Kanchana, A. Svane, S. Dugdale, and N. E. Christensen, Journal of Physics: Condensed Matter 25, 155501 (2013).

[28] V. S. Venkataraman, (private communication).

[29] D. Aoki, Y. Katayama, R. Settai, Y. Inada, Y. Ōnuki, H. Harima, and Z. Kletowski, Journal of the Physical Society of Japan 66, 3988 (1997).

[30] J. Xia, Y. Maeno, P. T. Beyersdorf, M. M. Fejer, and A. Kapitulnik, Phys. Rev. Lett. 97, 167002 (2006).

[31] J. Xia, E. Schemm, G. Deutscher, S. A. Kivelson, D. A. Bonn, W. N. Hardy, R. Liang, W. Siemons, G. Koster, M. M. Fejer, and A. Kapitulnik, Phys. Rev. Lett. 100, 127002 (2008).

[32] D. F. McMorrow, K. A. McEwen, U. Steigenberger, H. M. Rønnow, and F. Yakhou, Phys. Rev. Lett. 87, 057201 (2001).

\section{SUPPLEMENTAL MATERIAL}

\section{Eigenfunctions of the Luttinger Hamiltonian and their perturbations}

We first write the expression of energies and eigenfunctions of the Luttinger $k \cdot p$ Hamiltonian $\mathcal{H}_{0}$ presented in Eq.(1) and then describe the projection of $\mathcal{H}_{Q}^{(2)}$ into those eigenfunctions.

The two doublet eigenstates $\left(\psi_{1}, \psi_{2}, \psi_{3}, \psi_{4}\right)$ and their energies $\left(\epsilon_{1}, \epsilon_{1}, \epsilon_{2}, \epsilon_{2}\right)$ of Eq.(1) are described by,

$$
\begin{aligned}
& \epsilon_{1(2)}=-\frac{1}{4}\left(\left(4 c_{1}+5 c_{2}\right)\left(k_{x}^{2}+k_{y}^{2}+k_{z}^{2}\right) \pm 2 \sqrt{4 c_{2}^{2}\left(k_{x}^{4}+k_{y}^{4}+k_{z}^{4}-k_{y}^{2} k_{z}^{2}-k_{x}^{2} k_{z}^{2}-k_{x}^{2} k_{y}^{2}\right)+3 c_{3}^{2}\left(k_{y}^{2} k_{z}^{2}+k_{x}^{2} k_{z}^{2}+k_{x}^{2} k_{y}^{2}\right)}\right), \\
& \psi_{1}=\frac{1}{N}\left(\begin{array}{c}
a_{1}-i a_{2} \\
a_{3}+a_{4} \\
0 \\
a_{5}+i a_{6}
\end{array}\right), \quad \psi_{2}=\frac{1}{N}\left(\begin{array}{c}
-a_{5}+i a_{6} \\
0 \\
-a_{3}-a_{4} \\
a_{1}+i a_{2}
\end{array}\right), \quad \psi_{3}=\frac{1}{N}\left(\begin{array}{c}
0 \\
a_{5}-i a_{6} \\
-a_{1}+i a_{2} \\
-a_{3}-a_{4}
\end{array}\right), \quad \psi_{4}=\frac{1}{N}\left(\begin{array}{c}
a_{3}+a_{4} \\
-a_{1}-i a_{2} \\
-a_{5}-i a_{6} \\
0
\end{array}\right), \\
& a_{1}=2 \sqrt{3} c_{3} k_{x} k_{z}, \quad a_{2}=2 \sqrt{3} c_{3} k_{y} k_{z}, \quad a_{3}=c_{2}\left(k_{x}^{2}+k_{y}^{2}-2 k_{z}^{2}\right), \\
& a_{4}=2 \sqrt{4 c_{2}^{2}\left(k_{x}^{4}+k_{y}^{4}+k_{z}^{4}-k_{y}^{2} k_{z}^{2}-k_{x}^{2} k_{z}^{2}-k_{x}^{2} k_{y}^{2}\right)+3 c_{3}^{2}\left(k_{y}^{2} k_{z}^{2}+k_{x}^{2} k_{z}^{2}+k_{x}^{2} k_{y}^{2}\right)}, \\
& a_{5}=2 \sqrt{3} c_{2}\left(k_{x}^{2}-k_{y}^{2}\right), a_{6}=2 \sqrt{3} c_{3} k_{x} k_{y}, \\
& N=\sqrt{a_{1}^{2}+a_{2}^{2}+\left(a_{3}+a_{4}\right)^{2}+a_{5}^{2}+a_{6}^{2}} .
\end{aligned}
$$


Using eigenfunctinos $\psi_{1}, \psi_{2}, \psi_{3}$ and $\psi_{4}$, one can derive the projection of $\mathcal{H}_{Q}^{(2)}$ into those eigenfunctions,

$$
\begin{aligned}
& \tilde{\mathcal{H}}_{1}=\left\langle\psi_{1(2)}\left|\mathcal{H}_{Q}^{(2)}\right| \psi_{1(2)}\right\rangle=\vec{f}_{1}(\boldsymbol{k}) \cdot \vec{\sigma} \\
& \tilde{\mathcal{H}}_{2}=\left\langle\psi_{3(4)}\left|\mathcal{H}_{Q}^{(2)}\right| \psi_{3(4)}\right\rangle=\vec{f}_{2}(\boldsymbol{k}) \cdot \vec{\sigma}
\end{aligned}
$$

\section{Gyrotropic effect}

The gyrotropic coefficient is related to a transverse current proportional to the gradient of electric field: $j_{\mu}=$ $\lambda_{\mu \nu \gamma}^{G} d E_{\nu} / d x_{\gamma}$. Here, we consider an applied electric field $E_{\nu}\left(x_{\gamma}, t\right)=\operatorname{Re}\left[E e^{i\left(q x_{\gamma}-\omega t\right)}\right]$ and derive the expression of gyrotropic coefficient $\lambda_{\mu \nu \gamma}^{G}$. We note that this derivation is already studied in Ref.22. For a finite Berry curvature, the velocity of an electron wave packet with a band dispersion $\epsilon(\boldsymbol{k})$ is given by,

$$
\boldsymbol{v}(\boldsymbol{k})=\frac{1}{\hbar} \frac{\partial \epsilon(\boldsymbol{k})}{\partial \boldsymbol{k}}-\frac{e}{\hbar} \boldsymbol{E} \times \Omega(\boldsymbol{k}) .
$$

Based on Eq.(21), the local transverse current carried by electrons whose wave vectors lie in a slice of $k$-space of thickness $d k_{\gamma}$ is described by

$$
\begin{aligned}
J_{l o c}\left(k_{\gamma}, x_{\gamma}, t\right) & =-\frac{e^{2}}{\hbar} \frac{d k_{\gamma}}{4 \pi^{2}} E e^{i\left(q x_{\gamma}-\omega t\right)} \Phi\left(k_{\gamma}\right) \\
\Phi^{\gamma}\left(k_{\gamma}\right) & =\int_{o c c} d k_{\mu} d k_{\nu} \Omega^{\gamma}(\boldsymbol{k}) .
\end{aligned}
$$

The transverse nonlocal current, $J_{n l}\left(x_{\gamma}, t\right)$ is the summation of local current $J_{l o c}\left(k_{\gamma}, x_{\gamma}^{\prime}, t\right)$ for $x_{\gamma}>x_{\gamma}^{\prime}$ and $x_{\gamma}^{\prime}>x_{\gamma}$

$$
\begin{aligned}
& J_{n l}\left(k_{\gamma}, x_{\gamma}, t\right)=\frac{1}{l_{m f}} \int d x_{\gamma}^{\prime}\left(J_{l o c}^{x_{\gamma}^{\prime}>x_{\gamma}}+J_{l o c}^{x_{\gamma}>x_{\gamma}^{\prime}}\right) \\
& \int d x_{\gamma}^{\prime} J_{l o c}^{x_{\gamma}^{\prime}>x_{\gamma}}=\int_{x_{\gamma}}^{\infty} d x_{\gamma}^{\prime} p\left(x_{\gamma}-x_{\gamma}^{\prime}\right) J_{l o c}\left(k_{\gamma}, x_{\gamma}^{\prime}, t^{\prime}\left(x_{\gamma}^{\prime}\right)\right) \\
& =-\frac{e^{2}}{\hbar} \frac{d k_{\gamma}}{4 \pi^{2}} \Phi\left(k_{\gamma}\right) E \int_{x_{\gamma}}^{\infty} d x_{\gamma}^{\prime} \exp \left[-\frac{\left(x_{\gamma}^{\prime}-x_{\gamma}\right)}{l_{m f}}\right] \exp \left[i q x_{\gamma}^{\prime}-i \omega\left(t-\frac{\left(x_{\gamma}^{\prime}-x_{\gamma}\right)}{v_{F}}\right)\right] \\
& =-\frac{e^{2}}{\hbar} \frac{d k_{\gamma}}{4 \pi^{2}} \Phi\left(k_{\gamma}\right) E \int_{0}^{\infty} d \tilde{x}_{\gamma} \exp \left[-\frac{\tilde{x}_{\gamma}}{l_{m f}}+i q\left(\tilde{x}_{\gamma}+x_{\gamma}\right)-i \omega t+\frac{i \omega \tilde{x}_{\gamma}}{v_{F}}\right] \\
& =-\frac{e^{2}}{\hbar} \frac{d k_{\gamma}}{4 \pi^{2}} \Phi\left(k_{\gamma}\right) E e^{i\left(q x_{\gamma}-\omega t\right)} \frac{l_{m f}}{1-i \omega \tau-i q l_{m f}} \\
& \int d x_{\gamma}^{\prime} J_{l o c}^{x_{\gamma}>x_{\gamma}^{\prime}}=-\frac{e^{2}}{\hbar} \frac{d k_{\gamma}}{4 \pi^{2}} \Phi\left(k_{\gamma}\right) E \int_{-\infty}^{x_{\gamma}} d x_{\gamma}^{\prime} \exp \left[-\frac{\left(x_{\gamma}-x_{\gamma}^{\prime}\right)}{l_{m f}}\right] \exp \left[i q x_{\gamma}^{\prime}-i \omega\left(t-\frac{\left(x_{\gamma}-x_{\gamma}^{\prime}\right)}{v_{F}}\right)\right] \\
& =-\frac{e^{2}}{\hbar} \frac{d k_{\gamma}}{4 \pi^{2}} \Phi\left(k_{\gamma}\right) E \int_{0}^{\infty} d \tilde{x}_{\gamma} \exp \left[-\frac{\tilde{x}_{\gamma}}{l_{m f}}-i q\left(\tilde{x}_{\gamma}-x_{\gamma}\right)-i \omega t+\frac{i \omega \tilde{x}_{\gamma}}{v_{F}}\right] \\
& =-\frac{e^{2}}{\hbar} \frac{d k_{\gamma}}{4 \pi^{2}} \Phi\left(k_{\gamma}\right) E e^{i\left(q x_{\gamma}-\omega t\right)} \frac{l_{m f}}{1-i \omega \tau+i q l_{m f}} \\
& J_{n l}\left(x_{\gamma}, t\right)=-\frac{e^{2}}{\hbar} \frac{1}{(2 \pi)^{3}} E e^{i\left(q x_{\gamma}-\omega t\right)}\left(\int_{k_{\gamma}<0} d k_{\gamma} \frac{\Phi\left(k_{\gamma}\right)}{1-i \omega \tau-i q l_{m f}}+\int_{k_{\gamma}>0} d k_{\gamma} \frac{\Phi\left(k_{\gamma}\right)}{1-i \omega \tau+i q l_{m f}}\right) \\
& \approx \frac{e^{2}}{\hbar} \frac{2}{(2 \pi)^{3}} E e^{i\left(q x_{\gamma}-\omega t\right)} \int_{k_{\gamma}>0} d k_{\gamma} \Phi\left(k_{\gamma}\right) \frac{i q l_{m f}}{(1-i \omega \tau)^{2}}, \text { for } q l_{m f} \ll 1 \\
& J_{n l}\left(x_{\gamma}, t\right)=\lambda_{\mu \nu \gamma}^{G} i q E e^{i\left(q x_{\gamma}-\omega t\right)} \\
& \lambda_{\mu \nu \gamma}^{G}=\frac{e^{2}}{\hbar} \frac{2}{(2 \pi)^{3}} \frac{l_{m f}}{(1-i \omega \tau)^{2}} \int_{k_{\gamma}>0} d k_{\gamma} \Phi\left(k_{\gamma}\right), \text { for } q l_{m f} \ll 1
\end{aligned}
$$

within the relaxation time approximation $p\left(x_{\gamma}-x_{\gamma}^{\prime}\right) \propto$ $e^{-\left|x_{\gamma}-x_{\gamma}^{\prime}\right| / l_{m f}}$ with a mean free path $l_{m f}=v_{F} \tau$.
Now, we are interested in the derivation of Eq.(10) within the spherical Fermi surface approximation. The spherical coordinates are defined using radial, azimuthal and polar coordinates $(k, \varphi, \theta) ; \boldsymbol{k}=\left(k_{\mu}, k_{\nu}, k_{\gamma}\right)=$ $(k \sin \theta \cos \varphi, k \sin \theta \sin \varphi, k \cos \theta)$ 


$$
\begin{aligned}
\int_{k_{z}>0} d k_{z} \Phi\left(k_{z}\right) & =\int_{0}^{\infty} k^{2} d k \int_{0}^{\pi / 2} \sin \theta d \theta \int_{0}^{2 \pi} d \varphi \Omega_{\mathrm{occ}}^{\gamma}(k, \theta, \varphi) \\
& =\int_{0}^{\infty} k^{2} d k \int_{0}^{\pi / 2} \sin \theta d \theta \int_{0}^{2 \pi} d \varphi k^{-2} \sum_{i=1}^{2} \sum_{j= \pm} \Omega_{i, j}^{\gamma}(\theta, \varphi) n_{f}\left(E_{i, j}(k, \theta, \varphi)\right) \\
& \approx-2 \int_{0}^{\infty} d k \int_{0}^{\pi / 2} \sin \theta d \theta \int_{0}^{2 \pi} d \varphi\left(\Omega_{1,+}^{\gamma}(\theta, \varphi)\left|\vec{f}_{1}(k, \theta, \varphi)\right| \delta\left(\epsilon_{1}(k, \theta, \varphi)-\mu\right)\right. \\
& \left.\quad+\Omega_{2,+}^{\gamma}(\theta, \varphi)\left|\overrightarrow{f_{2}}(k, \theta, \varphi)\right| \delta\left(\epsilon_{2}(k, \theta, \varphi)-\mu\right)\right) \\
& \approx-\frac{1}{a} \frac{1}{W} \int_{0}^{\pi / 2} \sin \theta d \theta \int_{0}^{2 \pi} d \varphi\left(\Omega_{1,+}^{\gamma}(\theta, \varphi)\left|\vec{f}_{1}(\theta, \varphi)\right|+\Omega_{2,+}^{\gamma}(\theta, \varphi)\left|\vec{f}_{2}(\theta, \varphi)\right|\right)
\end{aligned}
$$

where $n_{f}(E)$ is the Fermi distribution with energy $E, \quad a$ is a lattice constant, four distinct energies are $E_{\ell, \pm}(\boldsymbol{k})=\epsilon_{\ell}(\boldsymbol{k}) \pm\left|\vec{f}_{\ell}(\boldsymbol{k})\right| . \quad\left(\vec{f}_{\ell}(\boldsymbol{k})\right.$ is defined in Eqs.(19) and (20).) We further labeled their distinct Berry curvatures as $\Omega_{\ell, \pm}(\boldsymbol{k})$, which satisfy $\Omega_{\ell, \pm}(\boldsymbol{k})=k^{-2} \Omega_{\ell, \pm}(\theta, \varphi)$ and $\Omega_{\ell,-}(\theta, \varphi)=-\Omega_{\ell,+}(\theta, \varphi)$. In the third step, we have used $n_{f}\left(E_{\ell, \pm}(\boldsymbol{k})\right) \approx n_{f}\left(\epsilon_{\ell}(\boldsymbol{k})\right) \mp\left|\vec{f}_{\ell}(\boldsymbol{k})\right| \delta\left(\epsilon_{\ell}(\boldsymbol{k})-\mu\right)$ for $W k \gg$ $V^{2} / \Delta \epsilon$. In the last step, we have assumed the spherical Fermi surfaces for both $\epsilon_{1(2)}$ with the wave vectors $k_{F}$ to separate the radial and angle integration, $\delta\left(\epsilon_{1(2)}(k, \theta, \varphi)-\mu\right) \approx$ $\frac{1}{W a^{2}} \delta\left(k^{2}-k_{F}^{2}\right)=\frac{1}{2 k_{F}} \frac{1}{W a^{2}}\left[\delta\left(k+k_{F}\right)+\delta\left(k-k_{F}\right)\right]$ and this leads to $\int_{0}^{\infty} d k(k a) \delta\left(\epsilon_{1(2)}(k, \theta, \varphi)-\mu\right)=1 /(2 a W)$ where $k a$ in the integration is from $f_{\ell}(k, \theta, \varphi)=(k a) f_{\ell}(\theta, \varphi)$.

Within the spherical Fermi surface approximation, we are left with the angle integration and realize that the integration of Berry curvature is not proportional to the Fermi wave vector $k_{F}$ in the limit of $W k \gg V^{2} / \Delta \epsilon$. Thus, the band split from the original doublet because of the perturbative quadrupolar order leads to the Berry curvatures, independent to the magnitude of Fermi wave vector but proportional to $V^{2} /(\Delta \epsilon W)$ only with azimuthal and polar angle dependence that captures broken inversion and mirror symmetries. 